\title{
Validation of Gamma Knife Perfexion Dose Profile Distribu- tion by a Modified Variable Ellipsoid Modeling Technique
}

\author{
Beong Ik Hur, Seong Jin Jin, ${ }^{2}$ Gyeong Rip Kim, ${ }^{3,4}$ Jong Hyeok Kwak, ${ }^{5}$ Young Ha Kim, ${ }^{4}$ Sang Weon Lee, ${ }^{3,4}$ Soon Ki Sung ${ }^{3,4}$ \\ Department of Neurosurgery, Pusan National University Hospital, Busan, Korea \\ Gamma-knife Center, ${ }^{2}$ Haeundae Paik Hospital, Inje University, Busan, Korea \\ Gamma-knife Center, ${ }^{3}$ Pusan National University Yangsan Hospital, Yangsan, Korea \\ Department of Neurosurgery, ${ }^{4}$ Pusan National University Yangsan Hospital, Yangsan, Korea \\ Department of Radiology, ${ }^{5}$ Pusan National University Yangsan Hospital, Yangsan, Korea
}

Objective : High precision and accuracy are expected in gamma knife radiosurgery treatment. Because of the requirement of clinically applying complex radiation and dose gradients together with a rapid radiation decline, a dedicated quality assurance program is required to maintain the radiation dosimetry and geometric accuracy and to reduce all associated risk factors. This study investigates the validity of Leksell Gamma plan (LGP)10.1.1 system of 5th generation Gamma Knife Perfexion as modified variable ellipsoid modeling technique (VEMT) method.

Methods : To verify LGP10.1.1 system, we compare the treatment plan program system of the Gamma Knife Perfexion, that is, the LGP, with the calculated value of the proposed modified VEMT program. To verify a modified VEMT method, we compare the distributions of the dose of Gamma Knife Perfexion measured by Gafchromic EBT3 and EBT-XD films. For verification, the center of an $80 \mathrm{~mm}$ radius solid water phantom is placed in the center of all sectors positioned at $16 \mathrm{~mm}, 4 \mathrm{~mm}$ and $8 \mathrm{~mm}$; that is, the dose distribution is similar to the method used in the $x, y$, and $z$ directions by the VEMT. The dose distribution in the axial direction is compared and analyzed based on Full-Width-of-Half-Maximum (FWHM) evaluation.

Results : The dose profile distribution was evaluated by FWHM, and it showed an average difference of $0.104 \mathrm{~mm}$ for the LGP value and $0.130 \mathrm{~mm}$ for the EBT-XD film.

Conclusion : The modified VEMT yielded consistent results in the two processes. The use of the modified VEMT as a verification tool can enable the system to stably test and operate the Gamma Knife Perfexion treatment planning system.

Key Words : Radiosurgery · Film dosimetry · Gamma knife · Perfection.

\section{INTRODUCTION}

In 1967, Lars Leksell introduced stereotactic radiosurgery in Sweden. In the 1990s, the Gamma Knife (GK) model B, the
Gamma plan, and a computerized workstation program were developed as a Gamma Knife Perfexion model by Gamma Knife 3rd generation model B, 4th generation C, and 4C. Currently, it is available as a 5th generation Gamma Knife Perfex-

- Received : June 26, 2020 •Revised : September 26, 2020 •Accepted : December 15, 2020

- Address for reprints : Soon Ki Sung

Department of Neurosurgery, Pusan National University Yangsan Hospital, Pusan National University School of Medicine, 20 Geumo-ro, Mulgeum-eup, Yangsan 50612, Korea Tel : +82-55-360-1522, Fax : +82-55-363-1915, E-mail : nscastle@naver.com, ORCID : https://orcid.org/0000-0001-7138-9621 
ion model. Sixth-generation icon models are developed ${ }^{7,10)}$.

An example of SRS is the popular and complete system Leksell Gamma Knife (LGK) (Elekta, Stockholm, Sweden) for radiosurgery ${ }^{9)}$. The narrow beam of each source used accumulates at the center of the radiation source to reach the target in the brain. The treatment is delivered by focusing several sources on the target. In particular, the LGK is a noninvasive surgical tool used to deliver highly conformal radiation to anatomically well-defined target lesions in the brain.

However, gamma knife radiosurgery (GKRS) is a significantly dangerous and fatal procedure because it administers high doses instantaneously, and the surgical procedure is completed in one day by irradiating high-energy gamma rays. Furthermore, it is important to maintain patient safety and precise treatment system for the preparation and treatment by GKRS because irradiation with a high dose of radiation is administered instantaneously. Therefore, regular quality management is essential to ensure accurate treatment planning and maintenance of a precise treatment system ${ }^{11)}$.

In a Gamma Knife, the target volume is precisely defined in three dimensions, and the dose distribution is consistent in the target volume and tolerance. In planning GKRS, the accuracy and reliability of the three-dimensional formula used to obtain the dose distribution of radiation surgery must be verified by actual measurements ${ }^{14)}$.

A representative and quality adjustment is the verification of the results of the Leksell Gamma plan (LGP) that is a treatment planning program provided by Elekta together with Gamma Knife equipment. A GKRS is performed based on the results of the LGP. There can be dose deviations owing to data corruption, unknown software limitations, and dose rate errors in treatment. Therefore, it is imperative to know the accuracy of the radiation dose delivery. Therefore, verification of the outcome of the plan is important. Quality assurance (QA) in GKRS is important and must be regular and continuous.

Existing methods for verifying LGP using several algorithms were reported by Tsai et al. ${ }^{15)}$, Marcu et al. ${ }^{12)}$, and Zhang et al. ${ }^{17)}$. However, these methods were available under a limited set of conditions. The method by Zhang et al. ${ }^{17)}$ can calculate treatment times that were accurate without plugs. Other methodologies were tested near the center of the target ${ }^{15)}$. Additionally, these studies had no uniform mathematical analysis. Instead of showing statistical consistency between the methods and LGP findings, only the size of errors were re- ported $^{13,15,17)}$.

In our previous study, to pioneer the addressing of these limitations, we developed a technique based on the known dose calculation method called the variable ellipsoid modeling technique (VEMT) and utilized in the GK model $4 C^{5)}$. In this study, we present a modified VEMT to fit the collimator cap of the cone shape of the GK Perfexion model. We modified the distance of 192 gamma beam paths from the cone shape to the collimator cap center axis through the modeled head and calculated the dose.

We verified the effectiveness of LGP10.1.1 system by comparing the modified VEMT to a calculated value from LGP10.1.1, and a measured value from EBT3 and EBT-XD film using the evaluation of the dose profile distribution by FWHM. Additionally, the verification method for the LGP10.1.1 was simultaneously validated by a model and a measurement that has not been ever attempted. We believe that the modified VEMT will be used as a reference for further QA adjustments and measurements following the maintenance of GK Perfexion based on this study.

\section{MATERIALS AND METHODS}

This study did not need IRB approval because it is not a study of patients. The method to model the shape of a patient's head and calculate the depth of several gamma-beam paths through a modeled head is essential in this model. The position of the radiation source and depth of penetration of the radiation through the scalp into the focal spot, the radiation dose measurement point, and the vertical distance between the radiation point and the radiation profile are calculated based on the underlying physics. In this study, we assume that the shape of a skull is an ellipsoid for the verification of the VEMT. We assume that the ellipsoid is centered on the mammillary body and the gamma beams are straightened to calculate the dose from the geometric relationship between the ellipsoid and the straight lines.

Gafchromic films are used as an advanced instrument for dosimetry systems. The radiochromic external beam therapy (EBT) film was released in 2004 by the International Specialty Products (Wayne, NJ, USA). In 2009, a second type of Gafchromic EBT film was developed. EBT2 films have a yellow marker dye in the active layer and a synthetic polymer as the 
binder component. In 2011, International Specialty Products launched the film generation EBT3 ${ }^{3)}$. In particular, Gafchromic EBT3 films are the new generation of Gafchromic films. An EBT3 film has a special polyester substrate that prevents the formation of Newton ring interference patterns in images from flatbed scanners. In addition, an EBT3 film is symmetric and eliminates the requirement to record the side of the film placed on the scanner. It is designed for the measurement of absorbed dose of ionizing radiation and has advantages of high spatial resolution and low energy dependence because it does not require post-treatment and darkroom operation after irradiation. However, the available dose range is 0.1 between 10 Gy. Therefore, EBT3 film is reported to be suitable for dose measurement of brachy-therapy, external beam radiotherapy, and the intensity-modulated radiotherapy technique ${ }^{13)}$.

Recently, a new type of radiochromic film, the EBT-XD film, was introduced for high-dose radiotherapy. Although the EBT-XD film has the same structure as the EBT3 film, it has a considerably different composition and a thinner active layer ${ }^{8)}$. Therefore, the EBT-XD film is improved with respect to film reading characteristics and shows advantages in the high-dose region for gamma beams.

\section{Coordinate system}

The coordinate systems used in the modified VEMT is the frame $\left(x_{f}, y_{f}, z_{f}\right)$, the shot center $\left(x_{s}, y_{s}, z_{s}\right)$, and frame center coordinate systems. There is a coordinate system $\left(x_{b}, y_{b}, z_{b}\right)$ in which each gamma beam is a $z_{b}$-axis, that is an axis of the coordinate system, and $x_{b} y_{b}$ is a plane perpendicular to the gamma beam axis through the focus. The coordinate relations of the coordinate systems are given by

$$
\begin{aligned}
& \left(\begin{array}{l}
x_{s} \\
y_{s} \\
z_{s}
\end{array}\right)=\left(\begin{array}{l}
x_{f}-s_{x} \\
-\left(y_{f}-s_{y}\right) \cos \alpha-\left(z_{f}-s_{z}\right) \sin \alpha \\
\left(y_{f}-s_{y}\right) \sin \alpha-\left(z_{f}-s_{z}\right) \cos \alpha
\end{array}\right) \\
& \left(\begin{array}{l}
x_{b} \\
y_{b} \\
z_{b}
\end{array}\right)=\left(\begin{array}{lll}
1 & 0 & 0 \\
0 & \cos \theta_{2} & \sin \theta_{2} \\
0 & -\sin \theta_{2} & \cos \theta_{2}
\end{array}\right) \cdot\left(\begin{array}{lll}
\cos \theta_{1} & \sin \theta_{1} & 0 \\
-\sin \theta_{1} & \cos \theta_{1} & 0 \\
0 & 0 & 1
\end{array}\right) \cdot\left(\begin{array}{l}
x_{s} \\
y_{s} \\
z_{s}
\end{array}\right)
\end{aligned}
$$

In Eq. (1), $\left(s_{x}, S_{y}, S_{z}\right)$ is the position coordinate of the shot focus from the frame coordinate system. In Eq. (2), $\theta_{1}$ and $\theta_{2}$ represent the azimuth and latitude, respectively of the source from the shot center coordinate system in the GK Perfexion model. In particular, Eq. (2) is a coordinate relation related to the dose profile distribution on the $x_{b} y_{b}$ plane that is introduced in the dose calculation.

\section{Model of the skull}

The modified VEMT was modeled as an ellipsoidal skull alternate to the method used in the LGP. To construct the ellipsoid, the lengths $\mathrm{a}, \mathrm{b}$, and $\mathrm{c}$ of the semi-principal axes can be measured on magnetic resonance imaging (MRI) images used for GKRS. The reference point of the mammillary body at the center of the skull was defined as the distance from the ellipsoid to the crown of the head, forehead, and temporal region. The ellipsoid is given by :

$$
\begin{aligned}
& \frac{\left[\left(x_{s}-l\right) \cos \beta+\left(y_{s}-m\right) \sin \beta \cos \alpha+\left(z_{s}-n\right) \sin \beta \sin \alpha\right]^{2}}{a^{2}} \\
& +\frac{\left[-\left(y_{s}-m\right) \sin \alpha+\left(z_{s}-n\right) \cos \alpha\right]^{2}}{c^{2}} \\
& +\frac{\left[-\left(x_{s}-l\right) \sin \beta+\left(y_{s}-m\right) \cos \beta \cos \alpha+\left(z_{s}-n\right) \cos \beta \sin \alpha\right]^{2}}{b^{2}}=1
\end{aligned}
$$

where $\left(x_{s}, y_{s}, z_{s}\right)$ is the coordinate from the shot center, $\alpha$ is the angle that the gamma angle $(\gamma)$ is related to, $\alpha=90-\gamma$ and $\beta$ is the angle of rotation of the image relative to the stereotactic frame in the MRI axial image. The point $(1, m, n)$ is the coordinate of the papillae in the frame coordinate system.

\section{Dose calculation of the VEMT}

The dose rates of the GK Perfexion model for a gamma beam at any point are calculated as follows ${ }^{16)}$. It is based on one gamma-beam calculation as shown in Eq. (4), and the doses at the target position are 192.

$$
\begin{aligned}
& \dot{D}_{i}\left(O_{c} ; t\right)=\frac{\dot{D}_{16}\left(80, t_{0}\right) \times O_{f}^{i}(c) \times e^{-\frac{\ln 2}{t_{1 / 2}\left(t-t_{0}\right)}} \times\left(\frac{d_{s f}^{i}}{d_{s f}^{i}-l^{i}}\right)^{2} \times e^{-\mu^{i}\left(d_{f}^{i}-80-l^{\prime}\right)}}{\times P^{i}\left(d_{i}=\frac{r_{i} \times d_{s c a l i n g}^{i}}{d_{\text {scaling }}^{i}-l^{i}}, \theta\right) \times\left(\sum_{i=1}^{5} n_{i} \times O_{f}^{i}(c=16 \mathrm{~mm})\right)^{-1}}
\end{aligned}
$$

The GK Perfexion model has $192 \times 3=576$ collimator channels that can be identified except when the source ${ }^{60} \mathrm{C}$ is positioned in the block position as shown in Fig. 1. For any position, owing to the gamma beam from each collimator, the radiation dose at the dose measurement position depends on the ring of the sector.

Therefore, although the dose distribution depends only on the distance at the $4 \mathrm{~mm}$ collimator position, at the $16 \mathrm{~mm}$ and $8 \mathrm{~mm}$ collimator positions the source is aligned with the collimator passages. Consequently, the dose profile distribu- 
tion has an asymmetric distribution $\left[\mathrm{D}\left(\mathrm{r}_{\mathrm{i}}, \theta\right) \neq \mathrm{D}\left(\mathrm{r}_{\mathrm{i}}, \theta\right)\right]$ depending on the distance and angle. In particular, the dose distribution value $P^{i}\left(d_{i}=\frac{r_{i} \times d_{\text {scaling }}^{i}}{d_{\text {scaling }}^{i}-l^{i}}, \theta\right)$ is determined according to the scaling distance value $d_{\text {scaling }}^{i}$, the vertical distance $\mathrm{r}_{\mathrm{i}}$ from the measurement point to the gamma beam axis, the rotation angle $\theta$ to the gamma beam axis, and the distance $l^{i}$ from the foot perpendicular to the focus from the measurement position to the gamma beam axis. Table 1 presents the respective values ${ }^{5}$.

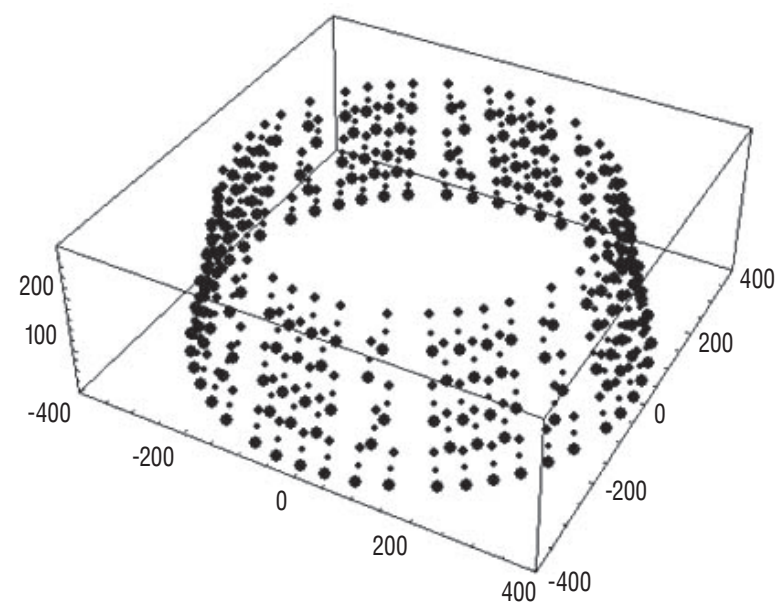

Fig. 1. Collimating system of GK Perfexion with 576 positions that radioisotopes can be positioned: $16 \mathrm{~mm}(-), 4 \mathrm{~mm}(-), 8 \mathrm{~mm} \mathrm{( \cdot )}$.

\section{Dose profile measurement by EBT3 and EBT-XD films}

The EBT3 and EBT-XD films were irradiated in the range of 0 to 40 Gy. The EBT3 film was irradiated until 10 Gy (the prescribed 50\% 5 Gy dose of maximum dose) and the EBT-XD film was irradiated until $40 \mathrm{~Gy}$ (the prescribed 50\% $20 \mathrm{~Gy}$ dose of maximum dose). The gamma beams were irradiated to the center of the $x-z$ and $x-y$ with 4,8 , and $16 \mathrm{~mm}$ collimators by the solid phantom (Elekta). After selecting one of the $x-z$ and $x-y$ directions of the solid-water phantom, as shown in Fig. 2A, the eight films on the center face were irradiated with a $16 \mathrm{~mm}$ collimator at $0,0.5,1,2,3,4,6$, and 8 Gy (EBT3) and $0,1,3,5,10,20,30$, and 40 Gy (EBT-XD) for the measurement of the calibration curve.

The irradiated EBT3 and EBT-XD films were scanned three times under the conditions of 24 bit color and 600 dpi using a scanner (Expression 11000XL; Epson, Suwashi, Japan), as shown in Fig. 2B and analyzed at the red and green channels using the Image program (image J, 64 bit 1.6.0 version; $\mathrm{Na}$ tional Institutes of Health, Bethesda, MD, USA). To calculate the standard deviation of fitting coefficient, OriginPro software (Originlab Corporation, Northampton, MA, USA) was used $^{1)}$ and the dose calibration curves were calculated.

Table 1. Parameters related to Gamma Knife Perfexion ${ }^{5)}$

\begin{tabular}{|c|c|c|c|c|}
\hline Collimator & $\begin{array}{c}\text { Output factor: } \\
O_{f}^{i}(c=4,8,16)\end{array}$ & $\begin{array}{l}\text { Attenuation coefficient }(1 / \mathrm{mm}) \text { : } \\
\mu^{i}\end{array}$ & $\begin{array}{l}\text { Scaling distances }(\mathrm{mm}): \\
d_{\text {scaling }}^{i}\end{array}$ & $\begin{array}{l}\text { Source to focus distance }(\mathrm{mm}) \text { : } \\
\qquad d_{s f}^{i}\end{array}$ \\
\hline P4_1 & 0.812 & 0.00685 & 387 & 482 \\
\hline P4_2 & 0.823 & 0.00682 & 390 & 474 \\
\hline P4_3 & 0.795 & 0.00688 & 397 & 489 \\
\hline P4_4 & 0.726 & 0.00693 & 408 & 521 \\
\hline P4_5 & 0.664 & 0.00689 & 430 & 545 \\
\hline P8_1 & 0.934 & 0.00652 & 384 & 405 \\
\hline P8_2 & 0.919 & 0.00656 & 392 & 411 \\
\hline P8_3 & 0.874 & 0.00658 & 404 & 424 \\
\hline P8_4 & 0.782 & 0.00666 & 418 & 459 \\
\hline P8_5 & 0.708 & 0.00658 & 443 & 475 \\
\hline P16_1 & 0.961 & 0.00695 & 391 & 467 \\
\hline P16_2 & 1 & 0.00688 & 389 & 449 \\
\hline P16_3 & 0.981 & 0.00689 & 393 & 453 \\
\hline P16_4 & 0.914 & 0.00701 & 399 & 485 \\
\hline P16_5 & 0.847 & 0.00707 & 419 & 518 \\
\hline
\end{tabular}




\section{RESULTS}

Precision is defined as a measure of the accuracy of the shape of the gamma beams and the intersection of the axes of these beams at a single point in space. We verify the modified VEMT by comparing the measured dose profiles of the dose distribution positioned in the volume surrounding the radio- logical focus position by EBT films and the calculated dose profiles by LGP10.1.1, assuming identical geometrical and radiophysical conditions. We consider the calculated results using the modified VEMT as the reference. The FWHM width at $50 \%$ height is the verification criterion for evaluating the steepness of the dose slope. The dose profiles of the dose distributions of the modified VEMT were compared using LGP
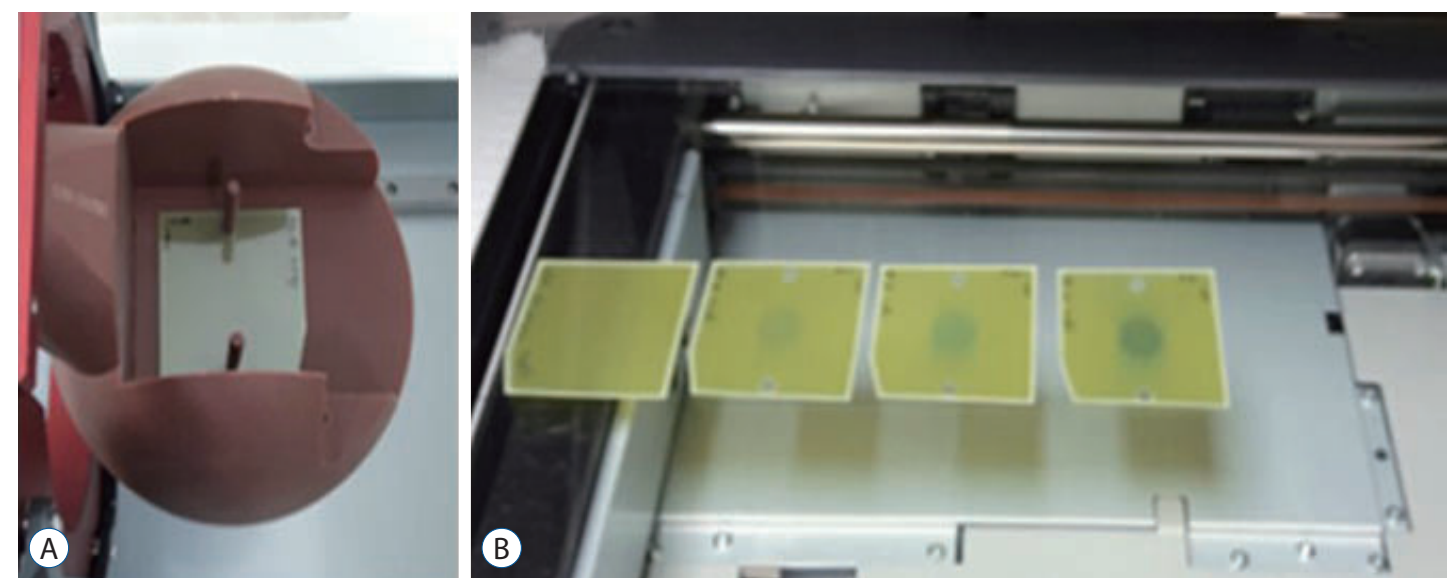

Fig. 2. Phantom and scanner for film measurement. A : Solid-water Phantom \& film. B : Epson Scanner.
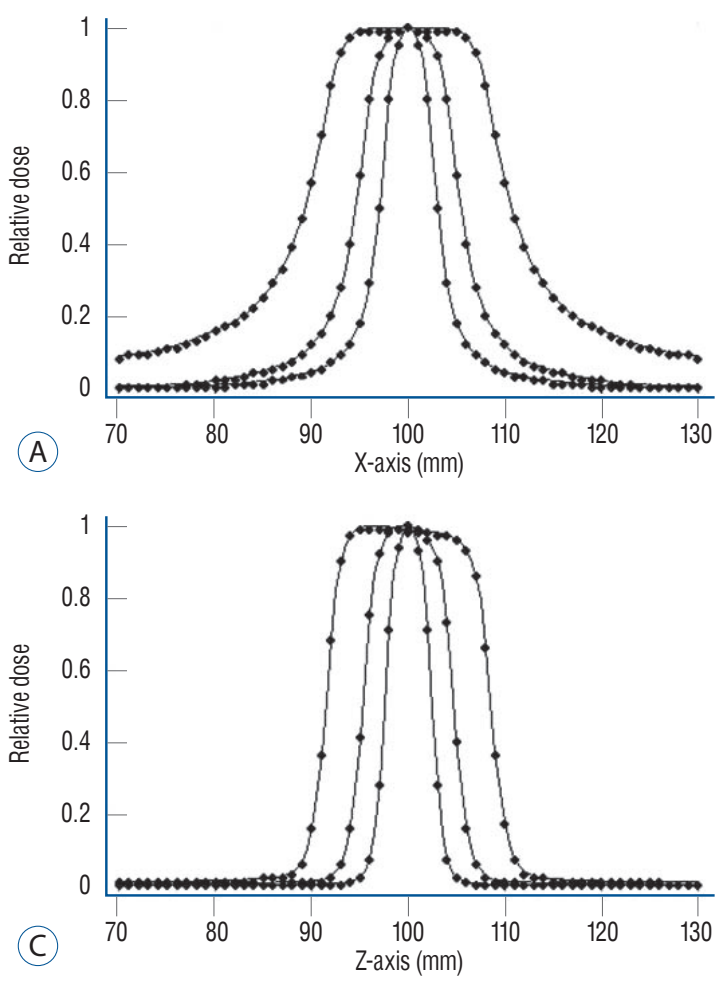

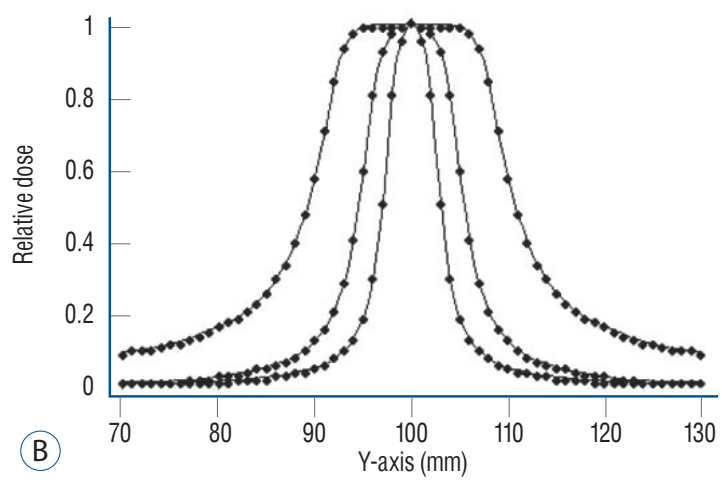

Fig. 3. The dose distribution obtained from the data extracted from LGP and the data calculated by the VEMT method are expressed using wolfram mathematica. Comparisons of dose profiles for the 4,8 , and $16 \mathrm{~mm}$ collimating configurations along the $x$ axis (A), the $y$ axis (B), and along the $z$ axis (C) using VEMT (-) and LGP (*). LGP : Leksell Gamma plan, VEMT : variable ellipsoid modeling technique. 
and EBT films.

\section{Validation of LGP 10.1.1 system}

The modified VEMT was evaluated by comparing to the results calculated by LGP10.1.1. Fig. 3 shows the dose profile distribution of VEMT and LGP10.1.1, and Table 2 presents the FWHM difference of the calculated values by the modified VEMT and LGP10.1.1 along the $\mathrm{x}, \mathrm{y}$, and $\mathrm{z}$ axes with 4, 8, and $16 \mathrm{~mm}$ collimators.

The difference in the FWHM of the proposed method was on an average $0.104 \mathrm{~mm}$ compared to that of the LGP10.1.1. Overall, the difference was less than $\pm 1 \mathrm{~mm}$; however, the difference in the $\mathrm{x}$ - and $\mathrm{y}$-axis values of the $16 \mathrm{~mm}$ collimator was largest at $0.25 \mathrm{~mm}$, as presented in Table 2 .

\section{Validation of the modified VEMT by EBT3 and EBT-XD films}

To evaluate the dose distributions of EBT3 and EBT-XD films, the dose calibration curves were initially measured by EBT films and calculated using an image. It was fitted with a quartic term-order polynomial curve $\left(\right.$ EBT3 $\mathrm{R}^{2}=9972$, EBT$\left.\mathrm{XD} \mathrm{R}^{2}=0.9984\right)$. We determined optical density data from the film gray scale value corresponding to $5 \mathrm{~Gy}$ and $20 \mathrm{~Gy}$, which is $50 \%$ the dose of 10 Gy and 40 Gy, respectively. Fig. 4 shows the dose calibration curve. The film gray scale corresponding to 5 Gy and 20 Gy of the dose calibration curve was 162 and 283 , respectively, using the fitted curve. The FWHM of the $\mathrm{x}$, $y$, and $z$ axes was calculated using the value obtained from the dose calibration curve.

Table 3 presents the difference in FWHM compared to the half-width reference value by the modified VEMT with mea-

Table 2. Differences of Full-Width-of-Half-Maximum obtained from LGP and VEMT on Gamma Knife Perfexion

\begin{tabular}{lccc}
\hline Collimator & VEMT $(\mathbf{m m})$ & LGP $(\mathbf{m m})$ & Difference $(\mathrm{mm})$ \\
\hline $4 \mathrm{~mm}$ (x-axis) & 6.10 & 6.16 & 0.06 \\
$4 \mathrm{~mm}$ (y-axis) & 6.10 & 6.16 & 0.06 \\
$4 \mathrm{~mm}$ (z-axis) & 5.16 & 5.04 & -0.12 \\
$8 \mathrm{~mm}$ (x-axis) & 11.10 & 11.06 & -0.04 \\
$8 \mathrm{~mm}$ (y-axis) & 11.10 & 11.06 & -0.04 \\
$8 \mathrm{~mm}$ (z-axis) & 9.71 & 9.8 & 0.09 \\
$16 \mathrm{~mm}$ (x-axis) & 22.00 & 21.75 & -0.25 \\
$16 \mathrm{~mm}$ (y-axis) & 22.00 & 21.75 & -0.25 \\
$16 \mathrm{~mm}$ (z-axis) & 17.41 & 17.44 & 0.03 \\
\hline
\end{tabular}

LGP : Leksell Gamma plan, VEMT : variable ellipsoid modeling technique
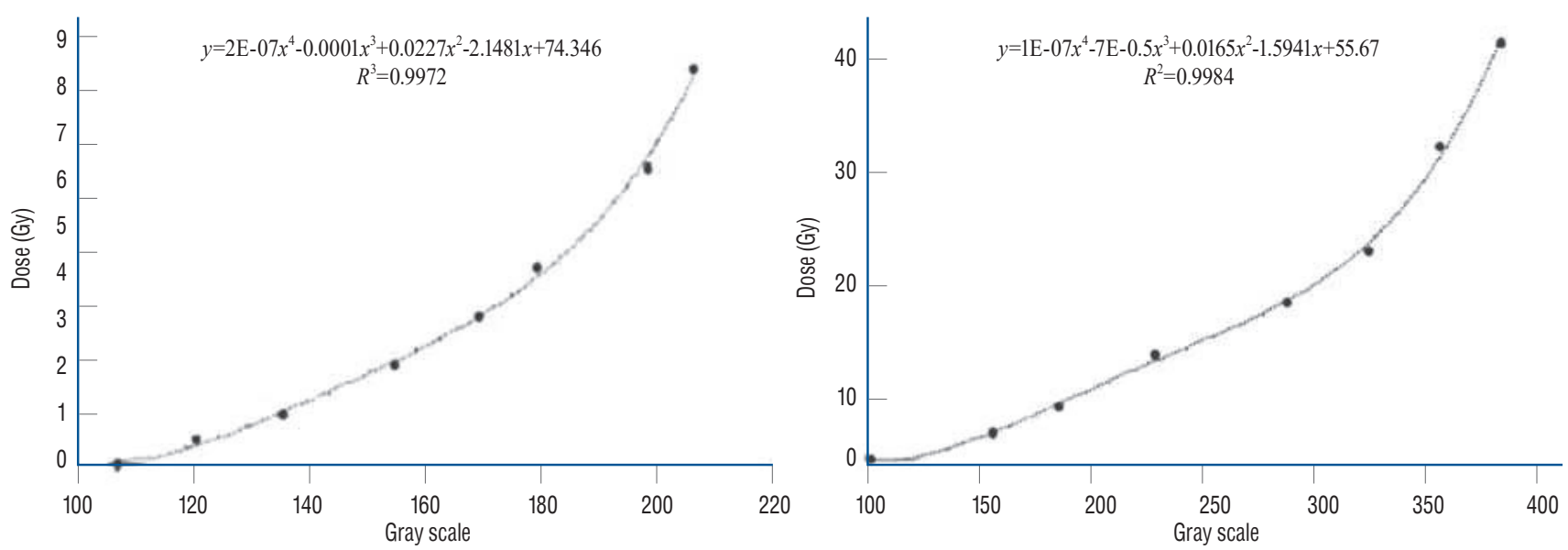

Fig. 4. Fitting does calibration curve at the red, green channel. 
sured values by EBT3, EBT-XD films at the $\mathrm{x}^{-}, \mathrm{y}^{-}$, and $\mathrm{z}$-axis of 4, 8, and $16 \mathrm{~mm}$ collimators of GK Perfexion. The difference in the FWHM was on an average $0.188 \mathrm{~mm}$ compared to that of the EBT3 film. Overall, the difference was less than $\pm 1 \mathrm{~mm}$; however, the difference in the $\mathrm{y}$-axis value of the $8 \mathrm{~mm}$ collimator was the largest $(0.415 \mathrm{~mm})$. In contrast, for the EBT-XD film, the difference in FWHM was $0.130 \mathrm{~mm}$. However, the difference in the $\mathrm{y}$-axis value of the $8 \mathrm{~mm}$ collimator was the

Table 3. Differences of Full-Width-of-Half-Maximum obtained from EBT3, EBT-XD films and VEMT on Gamma Knife Perfexion

\begin{tabular}{|c|c|c|c|c|}
\hline Collimator & VEMT (mm) & EBT3 film (mm) & EBT-XD film (mm) & D EBT-XD film Difference (mm) \\
\hline 4 mm (x-axis) & 6.10 & 6.223 & 6.203 & 0.103 \\
\hline 4 mm (y-axis) & 6.10 & 6.161 & 6.131 & 0.031 \\
\hline 4 mm (z-axis) & 5.16 & 5.341 & 5.241 & 0.081 \\
\hline 8 mm (x-axis) & 11.10 & 11.325 & 11.225 & 0.125 \\
\hline 8 mm (y-axis) & 11.10 & 11.515 & 11.482 & 0.382 \\
\hline 8 mm (z-axis) & 9.71 & 9.817 & 9.757 & 0.047 \\
\hline 16 mm (x-axis) & 22.00 & 21.618 & 21.690 & -0.310 \\
\hline 16 mm (y-axis) & 22.00 & 21.942 & 21.952 & -0.058 \\
\hline 16 mm (z-axis) & 17.41 & 17.442 & 17.432 & 0.032 \\
\hline
\end{tabular}

VEMT : variable ellipsoid modeling technique
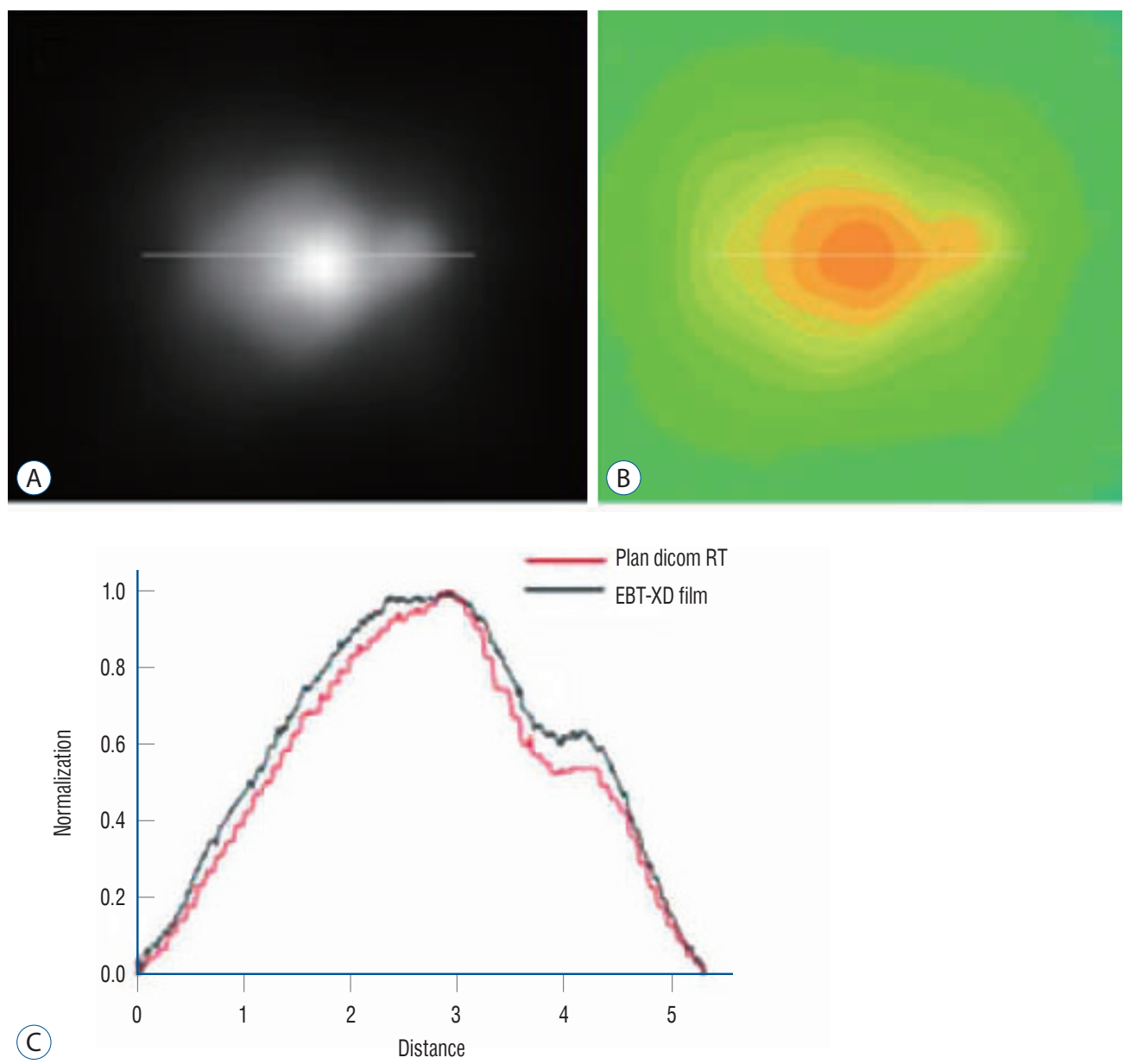

Fig. 5. Normalized intensity analysis (C) of the treatment plan (A) and the actual film survey (B) for a virtual target in the $x-y$ plane. 
largest $(0.382 \mathrm{~mm})$. Overall, the difference in FWHM was smaller than that of the EBT3 film for each collimator.

Consequently, the evaluation result of the EBT3 film showed a difference of $0.100 \mathrm{~mm}$ larger than the EBT-XD at a $\mathrm{z}$-axis value of $4 \mathrm{~mm}$ and an $\mathrm{x}$-axis value of $8 \mathrm{~mm}$, although the difference in the averages was $0.048 \mathrm{~mm}$. Although the EBT-XD film showed a smaller deviation than the EBT3 film, the difference was insignificant. From the evaluation, the EBTXD film showed a small deviation compared to that of the EBT3 film. Although the EBT-XD film showed a larger difference of $0.382 \mathrm{~mm}$ compared to that of the VEMT at the maximum value, the average difference was $0.130 \mathrm{~mm}$.

\section{Treatment plan evaluation by EBT-XD film}

Fig. 5 shows a design to treat the virtual $1 \mathrm{~mL}$ target at the center of the coordinate system and scan the EBT-XD film at the center of the $x-y$ plane after irradiating a prescription dose 20 Gy (50\% of maximum dose 40 Gy). From Fig. 5B, it can be observed that the film density is analyzed by the image $J$ that is compared to the concentration of RT file obtained from the treatment plan program in Fig. $5 \mathrm{~A}$ by the normalized values. From Fig. 5C, the 50\% dose position in the treatment plan and the $50 \%$ dose position in the EBT-XD film were qualitatively consistent. However, we cannot quantitatively compare the files of the treatment plan program with the EBT-XD film because there was no reference position to precisely match the centerline.

\section{DISCUSSION}

Generally, the treatment plan for GKRS must be verified cautiously because a high irradiation dose is administered between 20 Gy and 40 Gy instantaneously. It is related to maintaining patient safety. Therefore, performing periodic quality adjustment for safe treatment is essential. However, the actual LGP algorithm is unknown. This can introduce the challenge to design a dose verification method for GK treatment. To achieve an exact, safe and rigorous treatment, independent verification methods were developed by several groups ${ }^{2,8,12,17)}$. However, these studies did not addressed the calculation of the radiation depth for each gamma ray. We have developed a verification program called VEMT, a simulation program written in Java, to address this problem for the GK model $\mathrm{C}^{6}$.
In this study, as a verification tool, we proposed the modified VEMT for QA of the GK Perfexion model. For verification of LGP10.1.1, we used a modified VEMT and EBT3 and EBT-XD films for accurate measurement of the absorbed dose distribution. From the measured result, we evaluated the modified VEMT by using a FWHM.

Initially, an LGP and the modified VEMT were compared by the steepness of the dose gradient. From the evaluation, there was an insignificant difference in the collimator's FWHM values, as presented in Table 2. The average difference was $0.104 \mathrm{~mm}$. To evaluate the FWHM of the EBT3 and EBTXD films, the film gray scale corresponding to 5 Gy and 20 Gy from the dose calibration curve was selected. The dose calibration curve of EBT3 and EBT-XD films was developed by exposing films to known doses from 0 to 40 Gy using a gamma beam. We ignored the dosimetric and film reading characteristics, such as the film orientation effect and film sensitivity. The dose-response analysis was performed for the EBT3 and EBT-XD films using the red and green channels for accurate evaluation at high doses.

The calibration process for a set of films typically requires a significant time, effort, and care because several tens of films must be evaluated. Furthermore, the procedure must be repeated for sets of films with different numbers, even if they are of the same type. Therefore, it is desirable to develop a simple method of building a calibration curve for a new set of films using existing data without requiring repeating the calibration procedure ${ }^{4}$.

The comparison of the dose calibration curves for the different types of EBT film was performed by evaluating the FWHM by the modified VEMT. Studies considering the evaluation of films such as EBT3 and EBT-XD films are scarce ${ }^{13)}$. Although there is a small deviation in the FWHM, it shows no significant difference, as presented in Table 3. However, the comparison between the calculated and measured dose distributions of LGP10.1.1 and EBT films showed a significant difference.

For example, although the evaluation of the EBT-XD film showed a larger difference of $0.132 \mathrm{~mm}$ than that of the LGP10.1.1 at the maximum value, the average difference was $0.026 \mathrm{~mm}$. For the EBT-XD film, although the films were scanned thrice to minimize random noise and uncertainties using a flatbed film scanner, the difference in FWHM was an average of $0.026 \mathrm{~mm}$ compared to that of the LGP10.1.1. 
Therefore, we propose that the errors caused by the EBT film, from the process of scanning the film, and in the analysis using the image program can occur in the evaluation using the EBT film. Furthermore, the Newton's ring phenomenon occurred because of the foreign matter in the film. To prevent the occurrence of errors, it is necessary to establish a clearer reference position for the measurement position in film analysis using the image program. Furthermore, programming for image analysis is required.

The dose distributions of GK Perfexion model by VMET were consistent in the error range of the two verification methods. Accordingly, we can conclude that the validity of LGP10.1.1.is verified by the modified VEMT. As a result of this paper, our modeling actually confirmed possible as verification tool of model by evaluation of EBT films.

\section{CONCLUSION}

In this study, the validity of LGP10.1.1 was verified using the modified VEMT and EBT3 and EBT-XD films. The dose distributions in the $\mathrm{x}, \mathrm{y}$, and $\mathrm{z}$ axial directions were compared and analyzed by an FWHM evaluation. The results from the two verification methods were consistent. The modified VEMT maintained the dose profile distribution in 50\% line $\pm 1 \mathrm{~mm}$ by evaluation of EBT films. That is, the maximum inconsistency of the FWHM was less than $1 \mathrm{~mm}$ for all collimator configurations. Treatment verification using Gafchromic EBT films showed significant results as a verification tool through verification with the modified VEMT. Results from this study can be used as a reference for medical physicists that consider gamma knife perfexion. In particular, the EBT$\mathrm{XD}$ film is suited for dosimetric measurements in high-dose GKRS applications.

\section{CONFLICTS OF INTEREST}

No potential conflict of interest relevant to this article was reported.

\section{INFORMED CONSENT}

This type of study does not require informed consent.

\section{AUTHOR CONTRIBUTIONS}

\author{
Conceptualization : $\mathrm{BIH}$ \\ Data curation : SJJ \\ Formal analysis : JHK \\ Funding acquisition : GRK \\ Methodology : SWL \\ Project administration : SKS \\ Visualization : YHK \\ Writing - original draft : GRK \\ Writing - review \& editing : SKS
}

\section{ORCID}

$\begin{array}{ll}\text { Beong Ik Hur } & \text { https://orcid.org/0000-0001-5777-6019 } \\ \text { Seong Jin Jin } & \text { https://orcid.org/0000-0002-8859-7600 } \\ \text { Gyeong Rip Kim } & \text { https://orcid.org/0000-0003-4121-282X } \\ \text { Jong Hyeok Kwak } & \text { https://orcid.org/0000-0002-1910-3830 } \\ \text { Young Ha Kim } & \text { https://orcid.org/0000-0003-0477-3169 } \\ \text { Sang Weon Lee } & \text { https://orcid.org/0000-0002-3199-7072 } \\ \text { Soon Ki Sung } & \text { https://orcid.org/0000-0001-7138-9621 }\end{array}$

\section{- Acknowledgements}

This research was supported by the Korea Research Foundation (NRF) funded by the government (Ministry of Science, Technology and Information) in 2017 (NRF-2017R1C1B5077022).

\section{References}

1. Baghani HR, Aghamiri SM, Mahdavi SR, Robatjazi M, Zadeh AR, Akbari $M E$, et al. : Dosimetric evaluation of gafchromic EBT2 film for breast intraoperative electron radiotherapy verification. Phys Med 31 : 37-42, 2015

2. Beck J, Berndt A : An algorithm for independent verification of Gamma Knife treatment plans. Med Phys 31 : 2780-2784, 2004

3. Casanova Borca V, Pasquino M, Russo G, Grosso P, Cante D, Sciacero P, et al. : Dosimetric characterization and use of GAFCHROMIC EBT3 film 
for IMRT dose verification. J Appl Clin Med Phys 14 : 4111, 2013

4. Chung JP, Oh SW, Seong YM, Chun KJ, Chung HT : An effective calibration technique for radiochromic films using a single-shot dose distribution in Gamma Knife ${ }^{\circledR}$. Phys Med 32 : 368-378, 2016

5. Elekta : Leksell Gamma Knife Perfexion - Application Introductory Training, (Dose calculation for Leksell Gamma Knife Perfexion). Available at : https://www.elekta.com/radiosurgery/leksellgamma-knife-perfexion

6. Hur Bl, Choi BK, Sung SK, Cho WH, Cha SH, Choi CH : The variable ellipsoid modeling technique as a verification method for the treatment planning system of gamma knife radiosurgery. J Korean Neurosurg Soc 47 : 128-133, 2010

7. Hur BI : Comparisons between the two dose profiles extracted from Leksell GammaPlan and calculated by variable ellipsoid modeling technique. J Korean Soc Radiol 11 : 9-17, 2017

8. Jin JY, Drzymala R, Li Z : A simple method of independent treatment time verification in gamma knife radiosurgery using integral dose. Med Phys 31 : 3337-3344, 2004

9. Khachonkham S, Dreindl R, Heilemann G, Lechner W, Fuchs H, Palmans $H$, et al. : Characteristic of EBT-XD and EBT3 radiochromic film dosimetry for photon and proton beams. Phys Med Biol 63 : 065007, 2018

10. Kondziolka D, Maitz AH, Niranjan A, Flickinger JC, Lunsford LD : An evaluation of the Model $\mathrm{C}$ gamma knife with automatic patient position- ing. Neurosurgery 50 : 429-431; discussion 431-432, 2002

11. Maitz AH, Wu A, Lunsford LD, Flickinger JC, Kondziolka D, Bloomer WD : Quality assurance for Gamma Knife stereotactic radiosurgery. Int J Radiat Oncol Biol Phys 32 : 1465-1471, 1995

12. Marcu SM, Wu QJ, Pillai K, Weinhous MS : GammaPlan-Leksell Gamma Knife radiosurgery treatment planning verification method. Med Phys 27 : 2146-2149, 2000

13. Najafi M, Geraily G, Shirazi A, Esfahani M, Teimouri J : Analysis of Gafchromic EBT3 film calibration irradiated with gamma rays from different systems: Gamma Knife and Cobalt-60 unit. Med Dosim 42 : 159-168, 2017

14. Novotny J, Bhatnagar JP, Niranjan A, Quader MA, Huq Ms, Bednarz G, et al. : Dosimetric comparison of the Leksell Gamma Knife Perfexion and 4C. J Neurosurg 109 Suppl : 8-14, 2008

15. Tsai JS, Engler MJ, Rivard MJ, Mahajan A, Borden JA, Zheng Z : A formalism for independent checking of Gamma Knife dose calculations. Med Phys 28 : 1842-1849, 2001

16. Wu A, Lindner $G$, Maitz $A H$, Kalend AM, Lunsford LD, Flickinger JC, et al. : Physics of Gamma Knife approach on convergent beams in stereotactic radiosurgery. Int J Radiat Oncol Biol Phys 18 : 941-949, 1990

17. Zhang P, Dean D, Wu QJ, Sibata C : Fast verification of Gamma Knifetrade mark treatment plans. J Appl Clin Med Phys 1 : 158-164, 2000 